\title{
Zur geometrischen Optik der prismatischen Dispersion.
}

\author{
Von H. Opitz in Berlin.
}

Mit drei Abbildungen. (Eingegangen am 8. Juni 1922.)

Die geometrisch-optischen Untersuchnngen, betreffend die durch ein Prisma hervorgerufenen Dispersionserscheinangen, haben die Differentialformel

$$
d \varepsilon_{1}=\frac{\sin \varphi}{\cos \beta \cos \varepsilon_{1}} d n
$$

zur Grundlage, welche man aus den bekannten für ein Prisma geltenden Beziehungen

$$
\begin{aligned}
\sin \varepsilon & =n \sin \beta \\
\sin \varepsilon_{1} & =n \sin \beta_{1} \\
\varphi & =\beta+\beta_{1}
\end{aligned}
$$

leicht herstellen kann. Um eine Übersicht über die Erscheinungen zu bekommen, muß man aber den funktionalen Zusammenbang durchsichtiger gestalten, indem man eine passende unabhängige Veränderliche einführt. Es hat sich als zweckmäßig erwiesen, den Einfallswinkel $\varepsilon$ hierfür zu wählen, wodurch man die Zerstreuungsfunktion

$$
\pi(\varepsilon ; \varphi, n)=\frac{n \sin \varphi}{\Delta . \Delta_{1}}
$$

erhält, in welcher, den Veränderlichen $\varepsilon$ und $\varepsilon_{1}$, entsprechend,

$$
\Delta=\sqrt{n^{2}-\sin ^{2} \varepsilon} \text { and } \Delta_{1}=\sqrt{1-(\sin \varepsilon \cos \varphi-\Delta \cdot \sin \varphi)^{2}}
$$

zu setzen sind. Der Name für diese Funktion wird dadurch gerechtfertigt, daß das Integral

$$
\Delta \varepsilon_{1}=\int_{n_{1}}^{n_{2}} \pi(\varepsilon ; \varphi, n) d n
$$

den Zerstreunngswinkel zu ermitteln gestattet, welchen ein auf das Prisma fallender Strahl, der noch keine Dispersion erlitten hatte, nach der zweimaligen Brechung bildet ${ }^{1}$ ).

Die nähere Untersuchung der Eigenschaften dieser Funktion gibt ein vortreffliches Bild von dem Gange der Dispersionserscheinungen. Es empfiehlt sich aber, wegen der äußerst verwickelten Rechnnngen, welche die böheren Ableitungen der Funktion darbieten, bei der Diskussion neben der ersten Derivierten die graphische Darstellung zu Hilfe zu nebmen, indem man für die veränderlichen Parameter $n$ and $\varphi$ gegebene Werte in die Funktion eingehen läßt ${ }^{2}$ ).

1) Elster-Geitel-Festschrift, Brannschweig 1915, S. 307-312.

2) Verh. d. D. Phys. Ges. 17, 240-249, 1915. Dieser Vortrag ist vor Erscheinen der Festschrift gehalten worden. 
Die Vorteile dieses Verfabrens sind neuerdings von H. Erfle bestritten worden, indem er sich dabei anf den für den sogenannten kritischen Prismenwinkel durch eine leider unvollständige Induktion von mir erhaltenen falschen Wert stützt ${ }^{1}$ ). Es läßt sich jedoch zeigen, daß dieser kritische Winkel aus der Zerstreuungsfunktion ohne Benutzang einer kubischen Gleichung unmittelbar durch die erste Ableitung auf einfachere Art zu erhalten ist, als es bei Erfle geschehen ist.

Um den Verlauf der Zerstrenungsfunktion zu erkennen, bildet man zunächst

$$
\begin{gathered}
\frac{\partial \pi}{\partial \varepsilon}=\frac{n \sin \varphi}{2 \Delta^{3} \Delta_{1}^{3}}\left[\sin 2 \varepsilon\left\{1-n^{2} \sin ^{2} \varphi+\cos 2 \varphi\left(n^{2}-2 \sin ^{2} \varepsilon\right)\right\}\right. \\
\left.-\cos \varepsilon \sin 2 \varphi\left(n^{2}-4 \sin ^{2} \varepsilon\right) \cdot \Delta\right] .
\end{gathered}
$$

Man sieht dann sofort, daß diese Ableitung für alle Werte $\varepsilon$, welche der Bedingung $\varepsilon \leqq 0$ unterworfen sind, negativ sein muß, d.h. für diese Werte $\varepsilon$ muß die Zerstreuungskurve fallen, kann also kein Extremum haben 2). Es ist nämlich

$$
\left[\frac{\partial \pi}{\partial \varepsilon}\right]_{\varepsilon=0}=-\frac{n \sin \varphi \sin 2 \varphi}{2 \sqrt{1-n^{2} \sin ^{2} \varphi}}<0
$$

und aus der Beschaffenheit der $\pi$-Funktion ergibt sich weiter, daß für alle negativen Werte $\varepsilon$, d.h. für diejenigen Einfallswinkel, bei welchen der Einfallsstrahl mit der brechenden Kante des Prismas auf derselben Seite des Einfallslotes liegt, diese Funktion mit negativ wachsendem Argument bis ins Unendliche wächst, wenn

$$
\sin \varepsilon \cos \varphi-A \cdot \sin \varphi= \pm 1
$$

1) ZS. f. Instrkde. 39, 280-288 und 297-312, 1919.

2) Diese Diskussion erschien hier notwendig, weil H. Erfle in meinen Arbeiten Untersuchungen über das Vorzeichen des aus $\sin ^{2} \varepsilon$ hergeleiteten Einfallswinkels $\varepsilon$ vermißt hat. Ich verdanke den richtigen Wert $\varepsilon=45^{\circ} \mathrm{der}$ Festschrift (Note 1, S. 243) weder dem "Zufall", noch liegt der Fehler in der Berechnung des kritischen Prismenwinkels in meinem Vortrage (Note 2, S. 243) da, wo ihn Erfle gesucht hat [vgl. a. a. O. S. 286-287]. Die falsche Deutung der Gleichung

$$
\cos ^{3} \varphi+1 / 2 \cos ^{2} \varphi-1 / 2 \cos \varphi=\frac{n^{2}-1}{4 n^{2}}
$$

ist vielmehr die Folge eines fehlerhaften Induktionsschlusses meinerseits, verursacht durch Wertetabelle und graphische Darstellung. Diese Gleichung gibt für einen Wert $n$ einen Wert $\varphi$, für welchen das Minimum der Ablenkung einen Einfallswinkel $\varepsilon$ hat, der zugleich Lösung der kubischen Gleichung ist, welche sonst das Zerstreuungsminimum zu ermitteln gestattet (Verh. S. 246-247 als Beispiel). Erfle hat zwar meinen Fohler gefunden, die Ursache aber nicht. In den Verh. strtiche man im Text auf $\mathbf{8}$. 244 die letzten sieben Zeilen und beachte die Bemerkung in Fußnote 2 auf S. 243 dieser Mitteilung. 
wird, oder wenn für die absoluten Beträge

$$
|\sin \varepsilon|=\left|\cos \varphi-\sqrt{n^{2}-1} \cdot \sin \varphi\right|
$$

ist. Extremale Werte kann die Funktion also nur für positive $\varepsilon-W$ erte haben, oder

Maxima und Minima der Dispersion kann es nur für solche Einfallsstrablen geben, die durch das Einfallslot von der brechenden Kante des Prismas getrennt werden.

Dies wird durch die graphische Darstellung ${ }^{1}$ ) bestätigt (Fig. 1).

Wertetabelle zu Fig. 1. $n_{E}=1,785254$.

\begin{tabular}{|c|c|c|c|c|c|c|c|c|c|}
\hline$\varepsilon$ & $\varphi=10^{\circ}$ & $20^{0}$ & $30^{\circ}$ & $32^{0} 52^{\prime} 47^{\prime \prime}$ & $40^{\circ}$ & $45^{0} 4^{\prime} 39^{\prime \prime}$ & $50^{0}$ & $60^{\circ}$ & $68^{\circ} 7^{\prime} 54^{\prime \prime}$ \\
\hline 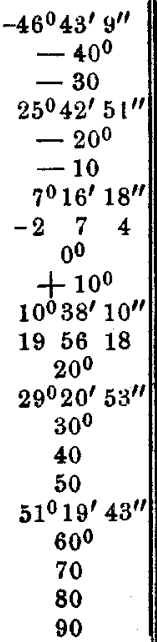 & $\begin{array}{c}\infty \\
0,481 \\
0,295 \\
\\
0,231 \\
0,199 \\
\\
0,183 \\
0,176 \\
\\
0,177 \\
0,184 \\
0,198 \\
0,218 \\
\\
0,244 \\
0,272 \\
0,296 \\
0,306\end{array}$ & $\begin{array}{c}\infty \\
0,893 \\
0,540 \\
\\
0,432 \\
0,384 \\
\\
0,363 \\
0,359 \\
0,367 \\
0,384 \\
0,405 \\
0,432 \\
0,451 \\
0,458\end{array}$ & $\begin{array}{l}1,109 \\
0,745 \\
0,625 \\
0,575 \\
0,558 \\
0,559\end{array}$ & $\begin{array}{c}\infty \\
2,203 \\
0,950 \\
\\
0,740 \\
\\
0,658 \\
0,625 \\
0,618 \\
\\
0,625 \\
0,639 \\
0,651 \\
0,657\end{array}$ & $\begin{array}{c}\infty \\
1,303 \\
0,963 \\
0,844 \\
0,797 \\
\\
0,782 \\
0,782 \\
0,787 \\
0,790\end{array}$ & $\begin{array}{c}\infty \\
18,268 \\
\\
1,448 \\
1,103 \\
0,981 \\
\\
0,931 \\
0,914 \\
0,909 \\
0,909\end{array}$ & $\begin{array}{c}\infty \\
6,398 \\
1,623 \\
1,267 \\
1,139 \\
1,086 \\
1,066 \\
1 ; 061\end{array}$ & $\begin{array}{c}\infty \\
6,369 \\
3,564 \\
2,848 \\
1,673\end{array}$ & $\infty$ \\
\hline
\end{tabular}

Die Kurven, welche bei gegebenem Brechungsindex $\boldsymbol{n}$ für verschiedene Prismenwinkel $\varphi$. erhalten werden, zeigen den Charakter von Funktionen dritten Grades, d.h. sie haben zwischen zwei extremalen Werten einen Wendepunkt. Die Extrema finden für diejenigen Werte $\varepsilon$ statt, welche den Gleichungen

$\cos \varepsilon=0$

$2 \sin \varepsilon\left(1-n^{2} \sin ^{2} \varphi+n^{2} \cos 2 \varphi-2 \sin ^{2} \varepsilon \cos 2 \varphi\right)$

$$
=\sin 2 \varphi\left(n^{2}-4 \sin ^{2} \varepsilon\right) \cdot \Delta
$$

1) Diese graphische Darstellung bildet eine Ergänzung und Verbesserung derjenigen auf S. 248 des B. 243, Note 2 genannten Vortrages. Der kritische Prismenwinkel $\bar{\varphi}$ würde, wenn nicht $n_{E}$, sondern $n_{B}=1,818597$ benutzt worden wäre, dem einzigen von E. Block in seiner Dorpater Dissertation vom Jahre 1873 (8. 41) angegebenen Werte $43^{\circ} 45^{\prime} 39^{\prime \prime}$ gleichkommen. 
H. Opitz,

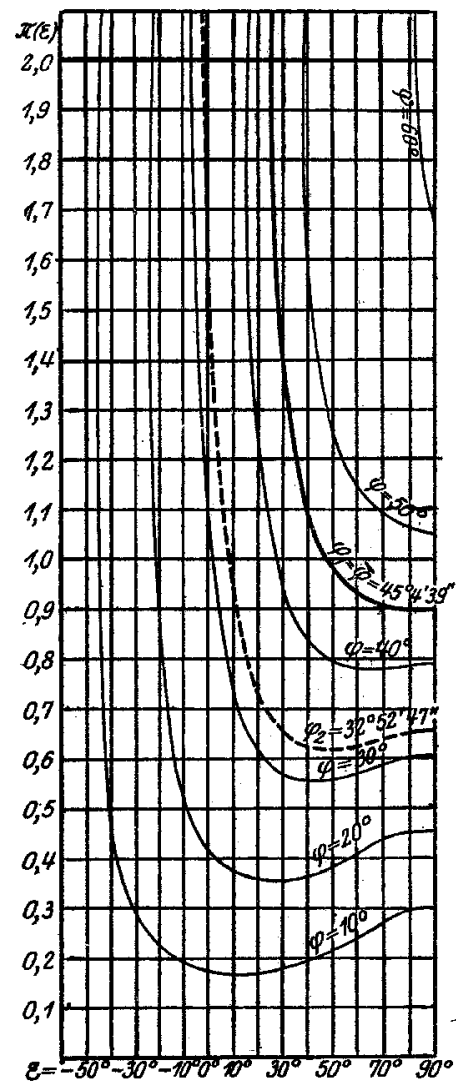

Fig. 1. Die Zerstrenungsfunktion für verschiedene Prismen aus Guynardschem Flintglase ( $\varepsilon$-Linie).

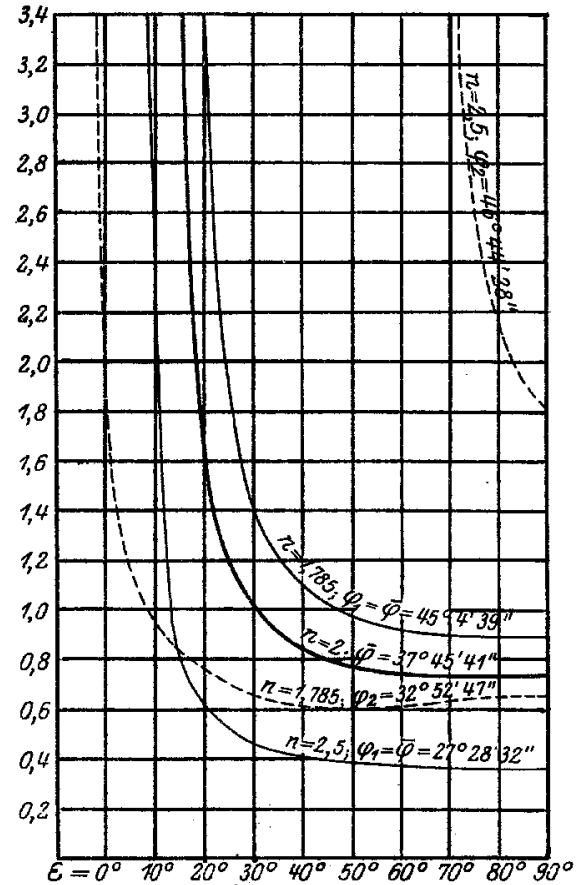

Fig. 3. Die Zerstreuungskurven für drei typische Fälle zu dem kritischen Prismen winkel.

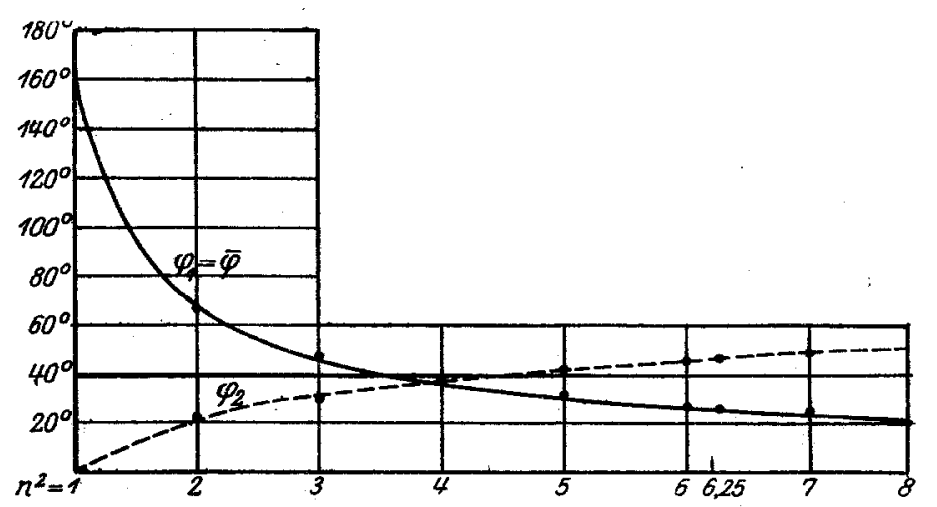

Fig. 2. Der kritisohe Prismenwinkel als Funktion von $n^{2}$. 
genügen. Während also für alle Kurven, die überhaupt Extrema haben, ein Maximum für $\varepsilon=90^{\circ}$ besteht, nähern sich mit wachsendem $\varphi$ für die Minima die $\varepsilon$-Werte dem erstgenannten, und bei $\varepsilon=90^{\circ}$ fallen die ausgezeichneten Punkte zusammen, wenn $\varphi$ denjenigen Wert erreicht hat, über den hinaus es keine Extrema für die Kurven mehr gibt, $\bar{\varphi}$ ist dann der kritische Prismenwinkel. Er läßt sich danach aus der Gleichung

$$
1-n^{2} \sin ^{2} \varphi+\left(n^{2}-2\right) \cos 2 \varphi=\sin \varphi \cos \varphi\left(n^{2}-4\right) \sqrt{n^{2}-1}
$$

berechnen.

Je nachdem man nun $\sin \varphi$ oder $\cos \varphi$ ausschlieBlich einführt, erhält man entweder oder

$$
n^{6} \sin ^{4} \varphi-\left(n^{2}-1\right)\left(n^{4}-2 n^{2}+8\right) \sin ^{2} \varphi+\left(n^{2}-1\right)^{2}=0
$$

$$
n^{6} \cos ^{4} \varphi-\left(n^{6}+3 n^{4}-10 n^{2}+8\right) \cos ^{2} \varphi+\left(2 n^{2}-3\right)^{2}=0 .
$$

Diese quadratischen Gleichungen für $\sin ^{2} \varphi$ and $\cos ^{2} \varphi$ liefern

und

$$
\sin ^{2} \varphi=\frac{n^{2}-1}{2 n^{6}}\left[n^{4}-2 n^{2}+8+\left(4-n^{2}\right) \sqrt{n^{4}+4}\right]
$$

$$
\cos ^{2} \varphi=\frac{n^{6}+3 n^{4}-10 n^{2}+8-\left(n^{2}-1\right)\left(4-n^{2}\right) \sqrt{n^{4}+4}}{2 n^{6}} .
$$

Durch Division würde man erhalten

und damit

$$
\operatorname{tg}^{2} \varphi=\frac{\left(n^{2}-1\right)\left(4-n^{2}+\sqrt{n^{4}+4}\right)^{2}}{4\left(2 n^{2}-3\right)^{2}}
$$

$$
\operatorname{tg} \varphi=\sqrt{n^{2}-1} \cdot \frac{4-n^{2}+\sqrt{n^{4}+4}}{2\left(2 n^{2}-3\right)} .
$$

Uber das Vorzeichen von $\sqrt{n^{4}+4}$ ist zunächst zu bemerken, daß in den Formeln für $\sin ^{2} \varphi$ und $\cos ^{2} \varphi$ die Zeichen wegen der Relation $\sin ^{2} \varphi+\cos ^{2} \varphi=1$ entgegengesetzt gewählt werden mußten. Da ferner

$$
n^{4}-2 n^{2}+8>\left(4-n^{2}\right) \sqrt{n^{4}+4}
$$

ist, so erhält man jedenfalls für $\sin \varphi$ zwei reelle Werte, denen zwei Winkel $\varphi_{1}$ und $\varphi_{2}$ entsprechen, je nachdem $+\sqrt{n^{4}+4}$ oder $-\sqrt{n^{4}+4}$ genommen wird. Von den obigen Formeln zur Berechnung von $\bar{\varphi}$ ist diejenige für $\sin ^{2} \varphi$ vorzuziehen, weil sie für die logarithmische Rechnung die bequemste ist. Hiernach ist $\sin ^{2} \varphi$ eine Funktion von $n^{2}$, welche für $n^{2}=1$ den Wert 0 , für $n^{2}=4$ den Wert 0,375 annir mt. Wenn $n^{2}$ weiter wächst, so folgt, da

$$
\lim _{n^{2}=\infty} \sin ^{2} \varphi=0 \text { oder } 1
$$


ist, je nachdem $\sqrt{n^{4}+4}$ positiv oder negativ genommen wird, daß die beiden $\varphi$-Kurven, nachdem sie sich im Punkte $\left(n^{2}=4 ; \varphi_{1}=\varphi_{2}\right.$ $=37^{\circ} 45^{\prime} 40^{\prime \prime}$ ) geschnitten haben, sich der Abszissenlinie $\varphi=0$ und der Geraden $\varphi=90^{\circ}$ asymptotisch nähern (Fig. 2).

Wertetabelle zu Fig. 2.

\begin{tabular}{|c|c|c|c|}
\hline$n^{2}$ & $n$ & $\varphi_{1}=\bar{\varphi}$ & $\varphi_{2}$ \\
\hline 1 & 1,00 & $180^{\circ}$ & $0^{0}$ \\
\hline 2 & 1,41 & $67^{0} 33^{\prime} 58^{\prime \prime}$ & $22^{0} 26^{\prime} 2^{\prime \prime}$ \\
\hline 3 & 1,73 & $\begin{array}{llll}47 & 20 & 55\end{array}$ & $\begin{array}{lll}31 & 33 & 20\end{array}$ \\
\hline 4 & 2,00 & $\begin{array}{lll}37 & 45 & 40\end{array}$ & $\begin{array}{lll}37 & 45 & 40\end{array}$ \\
\hline 5 & 2,24 & $\begin{array}{lll}32 & 3 & 55\end{array}$ & $\begin{array}{lll}42 \quad 22 & 12\end{array}$ \\
\hline 6 & 2,45 & $\begin{array}{lll}28 & 14 & 44\end{array}$ & $45 \quad 5740$ \\
\hline 6,25 & 2,50 & $\begin{array}{lll}27 & 28 & 32\end{array}$ & $\begin{array}{ll}46 & 44 \\
28\end{array}$ \\
\hline 7 & 2,65 & $\begin{array}{lll}25 & 28 & 48\end{array}$ & $\begin{array}{lll}48 & 51 & 26\end{array}$ \\
\hline
\end{tabular}

Allgemein wird der kritische Prismenwinkel in seiner Abhängig. keit von $n^{2}$ nur durch die $\varphi_{1}$-Kurve dargestellt, welche dem Ausdruck für $\sin ^{2} \varphi$ mit $+\sqrt{n^{4}+4}$ entspricht. Wenn $n^{2}<4$ ist, so ist $\varphi_{1}>\varphi_{2}$; dagegen ist $\varphi_{1}<\varphi_{2}$, wenn $n^{2}>4$ ist. Die kritische Kurve, die Zerstrenungskurve für $\bar{\varphi}$, ist immer die $\varphi_{1}$-Kurve, denn für Werte $n^{2}<4$ zeigt die $\varphi_{2}$-Kurve stets getrennte Zerstreungsmaxima und -minima, dagegen für $n^{2}>4$ überhaupt keine Extrema. Diese drei typischen Fälle werden für Guynandsches Flintglas $(n=1,785)$, für $n=2$ und für den Diamant $(n=2,5)$ in Fig. 3 dargestellt. Die einxige Kurve im Falle $n=2$ hat für $\varepsilon=90^{\circ} \mathrm{ihr}$ Minimum, ebenso wie die anderen $\bar{\varphi}$-Kurven.

Wertetabelle zu Fig. 3.

\begin{tabular}{|c|c|c|c|c|c|}
\hline$\varepsilon$ & $\begin{array}{c}n=2,5 \\
\bar{\varphi}_{1}=27^{\circ} 28^{\prime} 32^{\prime \prime}\end{array}$ & $\begin{array}{c}n=1,785 \\
\varphi_{2}=32^{\circ} 52^{\prime} 47^{\prime \prime}\end{array}$ & $\begin{array}{c}n=2 \\
\bar{\varphi}=37^{0} 45^{\prime} 41^{\prime \prime}\end{array}$ & $\begin{array}{c}n=1,785 \\
\frac{n}{\varphi_{1}}=4804^{\prime} 39^{\prime \prime}\end{array}$ & $\begin{array}{c}n=2,5 \\
\varphi_{2}=46^{\circ} 44^{\prime} 28^{\prime \prime}\end{array}$ \\
\hline $\begin{array}{l}90^{\circ} \\
80 \\
70 \\
60 \\
50 \\
40 \\
30 \\
20 \\
10 \\
0\end{array}$ & $\begin{array}{c}0,365 \\
0,365 \\
0,366 \\
0,370 \\
0,381 \\
0,411 \\
0,462 \\
0,612 \\
3,743 \\
\infty \\
\left(9^{0} 50^{\prime}\right)\end{array}$ & $\begin{array}{c}0,657 \\
0,651 \\
0,639 \\
0,625 \\
0,618 \\
0,625 \\
0,658 \\
0,740 \\
0,950 \\
2,203 \\
\infty \\
\left(-2^{0} 7^{\prime}\right)\end{array}$ & $\begin{array}{c}0,734 \\
0,735 \\
0,737 \\
0,748 \\
0,779 \\
0,852 \\
1,033 \\
1,770 \\
\infty \\
\left(15^{0} 39^{\prime}\right)\end{array}$ & $\begin{array}{c}0,909 \\
0,909 \\
0,914 \\
0,931 \\
0,981 \\
1,103 \\
1,448 \\
18,268 \\
\infty \\
\left(19^{0} 56^{\prime}\right)\end{array}$ & $\begin{array}{c}1,835 \\
2,211 \\
\infty \\
\left(71^{0} 59^{\prime}\right)\end{array}$ \\
\hline
\end{tabular}

Hiermit glaube ich denjenigen Teil seiner Kritik, in welchem H. Erfle sich in dankenswerter Weise um die Klarstellung der vorliegenden Aufgabe bemüht hat, genügend beleuchtet za haben. Wenn 
ich dennoch bei dieser Gelegenheit auf die weiteren Ausführungen meines Kritikers näher eingehe, so geschieht es, um unrichtige $\mathrm{Be}$ hauptungen in einigen wesentlichen Punkten auf ihr richtiges Maß zurückzuführen. So findet man z. B. bei H. Erfle folgenden Satz ${ }^{1}$ ): „Es ist somit bewiesen, daß die Anwendung einer kubischen Gleichung zur Bestimmung von $i_{1}$ (Opitz) oder auch von $i_{2}$ (Ditscheiner) überflüssig ist, da die Gleichung (12) viel schneller zu lösen ist, insbesondere wenn man die Näherungswerte (36) bzw. (37) benutzt."

Nan stellt er im Anschluß an (12) die Gleichung (30)

$$
\left(n^{2}-1\right) \operatorname{tg}\left(i_{1}^{\prime}-\alpha\right)=\operatorname{tg}\left(\alpha-2 i_{1}^{\prime}\right)
$$

auf, welche doch sicherlich in Beziehung auf $\operatorname{tg} i_{1}^{\prime}$ vom dritten Grade ist. Er löst sie nicht exakt, sondern approximativ, was natürlich bei jeder kubischen Gleichang möglich ist. Solche Methoden zur Löønng auszuschließen, hatte ich mir aber ausdrücklich vorgenommen ${ }^{2}$ ). Soviel über Abschnitt III der Erfleschen Arbeit.

Was den Abschnitt IV anbelangt, bei dem es sich in erster Linie um die größte Dispersion handelt, d.i. um den Fall $\pi(\varepsilon)=\infty$, so will ich zuvörderst auch hier zeigen, daß die diesen Abschnitt betreffenden Deduktionen die an den Anfang dieser Mitteilung gestellte Differentialformel und damit die Zerstreuungsfunktion zum Ausgangspunkt haben.

Nach der genannten Formel ist nämlich

$$
\cos \varepsilon_{1} d \varepsilon_{1}=\frac{\sin \varphi}{\cos \beta} d n=\frac{n \sin \varphi}{\sqrt{n^{2}-\sin ^{2} \varepsilon}} d n,
$$

wofür man auch setzen kann:

$$
d \sin \varepsilon_{1}=\sin \varphi \cdot d\left(\sqrt{n^{2}-\sin ^{2} \varepsilon}\right) .
$$

Integriert man zwisehen den die Randstrahlen des Zerstreuungswinkels bestimmenden Brechungsexponenten $n_{1}$ und $n_{2}$, wobei $n_{2}>n_{1}$ vorausgesetzt wird, d. h. bildet man

$$
\int_{\varepsilon_{1}}^{\varepsilon_{2}} d \sin \varepsilon_{1}=\sin \varphi \int_{n_{1}}^{n_{2}} d\left(\sqrt{n^{2}-\sin ^{2} \varepsilon}\right),
$$

1) a. a. O., S. 300. - Was die Bezeichnung der Größen betrifft, so ist zu setzen: $\quad a=\varphi, i_{1}=\varepsilon, i_{1}^{\prime}=\beta, i_{2}=\beta_{1}, i_{2}^{\prime}=\varepsilon_{1}$.

Es wäre erfreulich, wenn Erfle auf seine Anregung hin eine einheitliche Bezeichnung erreichen könnte. Man pfiegt aber jetzt wohl allgemein Winkelgrößen durch griechische Buchstaben zu bezeichnen. Weshalb Erfle, trotzdem er meine Arbeiten gekannt hat, für $\boldsymbol{\pi}(\varepsilon)$ das Funktionszeichen $\psi$ eingeführt hat, vermag ich nicht $\mathrm{za}$ beurteilen.

2) a. a. O., S. $241 \mathrm{der} V$ erh. 
indem man mit $\varepsilon_{1}$ und $\varepsilon_{2}$ die den Werten $n_{1}$ und $n_{2}$ zugehörenden Austrittswinkel bezeichnet, so erhält man

$$
\sin \varepsilon_{2}-\sin \varepsilon_{1}=\sin \varphi\left[\sqrt{n_{2}^{2}-\sin ^{2} \varepsilon}-\sqrt{n_{1}^{2}-\sin ^{2} \varepsilon}\right]
$$

wofür man noch setzen kann:

$$
\sin \frac{\varepsilon_{2}-\varepsilon_{1}}{2}=\frac{\sin \varphi}{2 \cos \frac{\varepsilon_{2}+\varepsilon_{1}}{2}}\left[\sqrt{n_{2}^{2}-\sin ^{2} \varepsilon}-\sqrt{n_{1}^{2}-\sin ^{2} \varepsilon}\right] .
$$

Diese Formeln bilden den Ausgangspunkt der Erfleschen Deduktionen, indem er, unter Benutzung der Reihenentwicklung nach Taylor für die Warzelgröße, Näherungsformeln aufatellt, die ihn sofort zu dem angeblich wichtigen Ergebnis führen, daß bei nahezu senkrechtem Austritt $\left(\cos \frac{\varepsilon_{2}+\varepsilon_{1}}{2}=1\right)$ die Dispersion der Differenz der Brechungsexponenten bei gegebenem $n$ und $\varphi$ proportional ist ${ }^{1}$ ).

Für die größte Dispersion $[\pi(\varepsilon)=\infty]$ hą nun $H$. Erfle nach dieser Methode eine Näherungsformel aufgegtellt, deren Rechnungsergebnisse er mit den von $\mathrm{E}$. Block berechneten Werten vergleicht, um den Wert der Formel festzustellen 2). Iob dagegen hatte gezeigt, wie mau mittels der Zerstreungaślunktion solche Größen verhältnismäßig einfach genau ermitteln kann. Das Integral ${ }^{3}$ )

$$
\Delta \varepsilon_{1}=\int_{n_{1}}^{n_{2}} \frac{n \sin x}{\Delta \cdot d_{l}} d n
$$

läßt sich dureh Einführung der Veränderichẹn

$$
\nu=\sin \varepsilon \cos \varphi-\Delta \cdot \sin \varphi
$$

an Stelle von $n$ sofort umformen in

$$
\Delta \varepsilon_{2}=\int_{\nu_{2}}^{\nu_{1}} \frac{d \nu}{\sqrt{1-\nu^{2}}}=\arcsin \nu_{1}-\arcsin \nu_{2} .
$$

1) Man vergleiche hiermit die Ergebnisse von A, Mousson, die mit sehr einfachen Mittein erzielt sind. - Die Physik auf Grundlage der Erfahrung. Zürich 1881 , II, 2, S. 418 .

2) a. a. $0 .$, S. 306/7.

3) Dieses Integral hat nicht den Beifall meines Kritikers gefunden. Er nennt e3 aber doch ndie zweckmäßig umgeťormto Gl. (4) (a. a. O., S. 288). Er hat nicht bedacht, dab es sich hier un ein funktionentheoretisches Problem handelt, weiches - technisch ansgedrückt - zu einer independenten Darstellung: für den Zerstreuunggwinkel führen sollte und geführt hat. Unter Berücksichtigung cer Bemerkung in TuBnote 2 auf $\$ .243$ ist es wohl klar, dab die wirkliche Bedeutung der neu eingeführten Veränderlichen $\nu$ nicht besonders hervorgehoben zu werden brauchte. Der gerügte Druckfehler besteht übrigens nicht, da Vertauschung der Grenzen eines bestimmten Integrals Vorzeichenänderung nach sich zieht. 
Im Falle $\pi(\varepsilon)=\infty$ ist nan $v=-1$. Die Randistrablev sollen die Brechungsexponenten $n_{2}=n_{H}=1,818597$ tnd $n_{1}=n_{E}=1,769702$ baben. Der Fall $\varphi=40^{\circ}$, in weichem die Zerstreuungswinkel bei Frfle und Bleck den größten Unterschied zeigten, soll hier durchgerechnet werden. Den Kinfallswinkel $\varepsilon$ findet man aus der Gleichung

$$
\sin \varepsilon \cos \varphi-\Delta \cdot \sin \varphi=v_{2}=-1
$$

durch Auflösung nach $\sin \hat{c}$ :

$$
\sin \varepsilon=-\cos \varphi+\sin \varphi \cdot \sqrt[3]{n^{2}-1} .
$$

Man erbält für $n_{H}$ and $\varphi=40^{\circ}$ : $\sin \varepsilon=0,2103357, \varepsilon=12^{\circ} 8^{\prime} 31^{\prime \prime}$. Jetzt berechnet man $\nu_{1}=\sin \varepsilon \cos \varphi-A \cdot \sin \phi=0,161127-1,1294.79$, indem man in $\Delta$ für $n$ die Größe $n_{2}$ einsetzt. Es folgt $v_{1}=-0,968352$. Mithin ist

$$
\begin{aligned}
\Delta \varepsilon_{1} & =-\arcsin 0,968352+\arcsin 1 \\
& =90^{\circ}-75^{\circ} 32^{\prime} 48^{\prime \prime}=14^{\circ} 27^{\prime} 12^{\prime \prime} .
\end{aligned}
$$

Block batte gefunden $\varepsilon=12^{\circ} 6^{\prime} 50^{\prime \prime}, \Delta \varepsilon_{1}=14^{\circ} 21^{\prime} 35^{\prime \prime}$ and ist von Erfle verbessert worden za $\varepsilon=12^{\circ} 8^{\prime} 26^{\prime \prime}, \Delta \varepsilon_{1}=14^{\circ} 27^{\prime} 0^{\prime \prime}$. Dagegen hat Erfile durch seice Näherungsformel $\Delta \varepsilon_{1}=14^{\circ} 26^{\prime} 15^{\prime \prime}$ ermittelt, was gegen den oben genan berechneten Wert eine Differenz von 57" ausmacht. Und die Rechnung nach der Erfleschen Näherungsformel ist wohl kaum einfacher, als die soeben durchgeführte.

Auf weitere Beispiele will ich nicht eingehen, sondern erlaube mir, auf die am Schluß meines Vorrages angefihhrten Rechnungen zu verweisen, wo auch der Fall des streifenden Ein- und Austritts der Strahlen erwähnt ist, den $H$. Frfle ebenfalls nur unter Benutzung der Zerstreuungsfunition bat behandeln können.

Zasammenfassung. 1. Die in meinen Arbeiten über das Minimum Rer Dispersion angestrebte übersichtliche funktionentheoretische Behandlung batte einen Fehler aufzuweisen, welcher den sogenannten kritischen Prismenwinkel betraf, $a$ i. den Winkel, der - wenn er überschritten wird - keine Extrema für die Dispersion mebr zuläßt. Es wird der möglichst einfache Weg zur Bestimmung dieses Grenzwinkels gegeben.

2. Die cankenswerte Kritik, welche H. Erfle zur Entdeckung des Fehlers geführt hat, wird darch Besprechnng einiger Beispiele auf ibr richtiges $\mathrm{Maß}$ zurückgeführt, und es wird gezeigt, daß die prismatischen Dispersionserscheinungen die beste Grundlage in einer Differentialformel und in der sogenannten Zerstreuungsfunktion haben.

Berlin, im Mai 1922. 